NBER WORKING PAPER SERIES

THE INFORMATION PHARMS RACE AND COMPETITIVE DYNAMICS OF PRECISION MEDICINE: INSIGHTS FROM GAME THEORY

\author{
Ernst R. Berndt \\ Mark R. Trusheim \\ Working Paper 24020 \\ http://www.nber.org/papers/w24020
NATIONAL BUREAU OF ECONOMIC RESEARCH
1050 Massachusetts Avenue
Cambridge, MA 02138
November 2017

We thank participants in the September 13-14, 2017 National Bureau of Economic Research Conference on Economic Dimensions of Personalized and Precision Medicine in Santa Monica, California. This research was not sponsored. The views expressed herein are those of the authors and do not necessarily reflect the views of the National Bureau of Economic Research.

NBER working papers are circulated for discussion and comment purposes. They have not been peer-reviewed or been subject to the review by the NBER Board of Directors that accompanies official NBER publications.

(C) 2017 by Ernst R. Berndt and Mark R. Trusheim. All rights reserved. Short sections of text, not to exceed two paragraphs, may be quoted without explicit permission provided that full credit, including $(\odot)$ notice, is given to the source. 
The Information Pharms Race and Competitive Dynamics of Precision Medicine: Insights from Game Theory Ernst R. Berndt and Mark R. Trusheim

NBER Working Paper No. 24020

November 2017

JEL No. I11,L13,L65

\begin{abstract}
Precision medicines inherently fragment treatment populations, generating small-population markets, creating high-priced "niche busters" rather than broadly prescribed "blockbusters". It is plausible to expect that small markets will attract limited entry in which a small number of interdependent differentiated product oligopolists will compete, each possessing market power. Multiple precision medicine market situations now resemble game theory constructs such as the prisoners' dilemma and Bertrand competition. The examples often involve drug developer choices created by setting the cut-off value for the companion diagnostics to define the precision medicine market niches and their payoffs. Precision medicine game situations may also involve payers and patients who attempt to change the game to their advantage or whose induced behaviors alter the payoffs for the developers. The variety of games may predictably array themselves across the lifecycle of each precision medicine indication niche and so may become linked into a sequentially evolving meta-game. We hypothesize that certain precision medicine areas such as inflammatory diseases are becoming complex simultaneous multi-games in which distinct precision medicine niches compete. Those players that learn the most rapidly and apply those learnings the most asymmetrically will be advantaged in this ongoing information pharms race.
\end{abstract}

\author{
Ernst R. Berndt \\ MIT Sloan School of Management \\ 100 Main Street, E62-518 \\ Cambridge, MA 02142 \\ and NBER \\ eberndt@mit.edu \\ Mark R. Trusheim \\ Massachusetts Institute of Technology \\ Alfred P. Sloan School of Management \\ 100 Main Street, E62-527 \\ Cambridge, MA 02142 \\ mtrusheim@cobioconsulting.com
}




\section{Introduction to Precision Medicines}

Over the last decade advances in our understanding of the human genome and biology more generally have facilitated the development and commercialization of therapies that, when combined with some form of biomarker diagnostic, are able to identify subpopulations of patients that are likely to respond differentially to the therapy - either positively or negatively. This combination of biomarker and therapy has been given a variety of names - e.g., personalized medicine, precision medicine, tailored medicine, and stratified medicine. ${ }^{1,2,6}$ The majority of these medicines identify multi-person subpopulations. Truly personalized medicines extract and harvest human tissues, expose them to external treatment, and then infuse or inject them back into the patient. The FDA recently approved a new gene therapy, CTL019 (tisagenlecleucel) CAR-T cell therapy developed by Novartis for B-cell acute lymphoblastic leukemia (ALL) - a truly personalized medicine. ${ }^{3}$ Prior to that, the only FDA approved personalized medicines were Provenge from Dendreon (recently again independent) ${ }^{4}$ and a variety of cord blood transplant products. ${ }^{5}$

Regardless of what one calls it, the combination of a therapy and a companion diagnostic $(C D x)$ relies critically on the ability ex ante to distinguish treatment responders from non-responders. The precision medicine opportunity arises because many drugs prove efficacious for only some who take them. For example, clinical remission rates for tumor necrosis factor alpha (TNF $\alpha$ ) inhibitors in auto-immune diseases such as Crohn's disease and ulcerative colitis are approximately $25-40 \%{ }^{7}$ For many oncology therapeutics perhaps only $20-$ $30 \%$ respond and have their life expectancy (months of overall survival) increased. ${ }^{8}$ 
Like drugs, however, no diagnostic is perfect. The performance of a diagnostic is quantified by metrics such as sensitivity (the portion of true positives that are diagnosed as such) and specificity (the portion of true negatives that receive a negative diagnostic result). For example, the HER2 test used to qualify breast cancer patients for receiving trastuzumab (Herceptin) was shown to be approximately $89 \%$ sensitive and only $83 \%$ specific. The reported performance also indicated that a patient testing positive had a 39\% ( 2 out of 5 ) chance of responding to trastuzumab-the positive predictive value of the companion diagnostic.9,10

\section{Precision Medicine Often Results In Oligopolies}

Companion diagnostics inherently reduce the size of the potentially treated population by identifying sub-populations. In practice, precision medicine indications often possess relatively small numbers of patients. For scientific feasibility reasons, already small indications such as cancer have seen the most precision medicine products and so become even more fragmented. ${ }^{11,12}$ This combination of small patient populations and high mortality conditions has resulted in precision medicines becoming high priced "niche busters" rather than broadly prescribed "block busters" such as blood pressure medicines.

One might expect small markets to attract few entrants. In fact, this is largely the case. A study of oncology products (both approved and in development) showed that the number of competitors with products for precision medicine drug targets is generally under five, with one or two exceptions such as HER2 and EGFR targeted therapies. ${ }^{13}$ Precision medicines to date generally involve a small number of interdependent differentiated product oligopolists, each having some market power. This brings us to game theory. 


\section{Game Theory Useful in Oligopolistic Situations}

In markets with a small number of firms producing slightly differentiated products, one firm's pricing, output and quality decisions affect all other firms' similar decisions, and vice versa. In a cooperative game, players (firms) can negotiate binding contracts that allow them to plan joint strategies. In many countries, cooperative games are prohibited by law, and enforced by anti-trust or anti-combines authorities. We will not discuss such games here. Rather, we will focus on non-cooperative games in which the negotiation and enforcement of binding contracts are not possible, yet players can anticipate, observe and react to others' behaviors.

An example non-cooperative game is a situation in which two competing firms take each other's likely behavior into account when independently setting prices, or making R\&D decisions. A strategy is a rule or plan of action for playing the game to maximize the payoff. If firms solely seek profits, an optimal strategy for a player is the one that maximizes their expected profit (or net present value). In addition to assuming profit maximization as a firm's only objective, game theorists typically assume players are rational. In game theory this means that firms think through the consequences of their actions, with each one asking, "Since our competitors are rational and act to maximize their own expected profits, what will they do and how should we take their likely behavior into account when making our decisions?"14

\section{Plan of Paper}

Game theory has useful insights for traditional drug development in cases where emerging drug classes contain few products. Here we introduce the additional complications 
that arise when firms choose to use a companion diagnostic to stratify patients into subpopulations. In this paper, we will focus on markets for precision medicines that frequently include game theory situations. We are not game theorists. Our goal here is to provide examples of precision medicine market developments that illustrate a variety of classic games and variations, beginning with the iconic prisoners' dilemma game that occurs in the situation of selecting a companion diagnostic cut-off value. But before we begin discussing the games we briefly describe the key features of precision medicine that underpin the rules and payoffs of the games.

\section{Games People Play When Precision Medicine Is Not Precisely Accurate}

Companion diagnostics have been used in oncology since at least the 1990s when trastuzumab (Herceptin) was launched with a companion diagnostic for HER2 overexpression. ${ }^{15}$ Since then, many stratified medicines have been introduced not only into oncology but also into fields such as infectious disease (sofosbuvir; Sovaldi and HCV genotypes 1-4), respiratory disease (omalizumab; Xolair and IgE levels for both patient selection and dosing), and neurodegenerative disease (natalizumab; Tysabri) and John Cunningham Virus ([JCV] testing). ${ }^{11,15}$

A firm may choose to develop a companion diagnostic, or not. But when it does, by setting the companion diagnostic cut-off value, developers link science, the clinic and the marketplace to create a precision medicine. Selecting the cut-off value connects scientific understanding of both therapeutic response and biomarker performance to change the 
observed efficacy in the selected clinical trial population. This in turn has implications for pricing, especially when price is linked to patient benefit as in "value pricing" regimes.

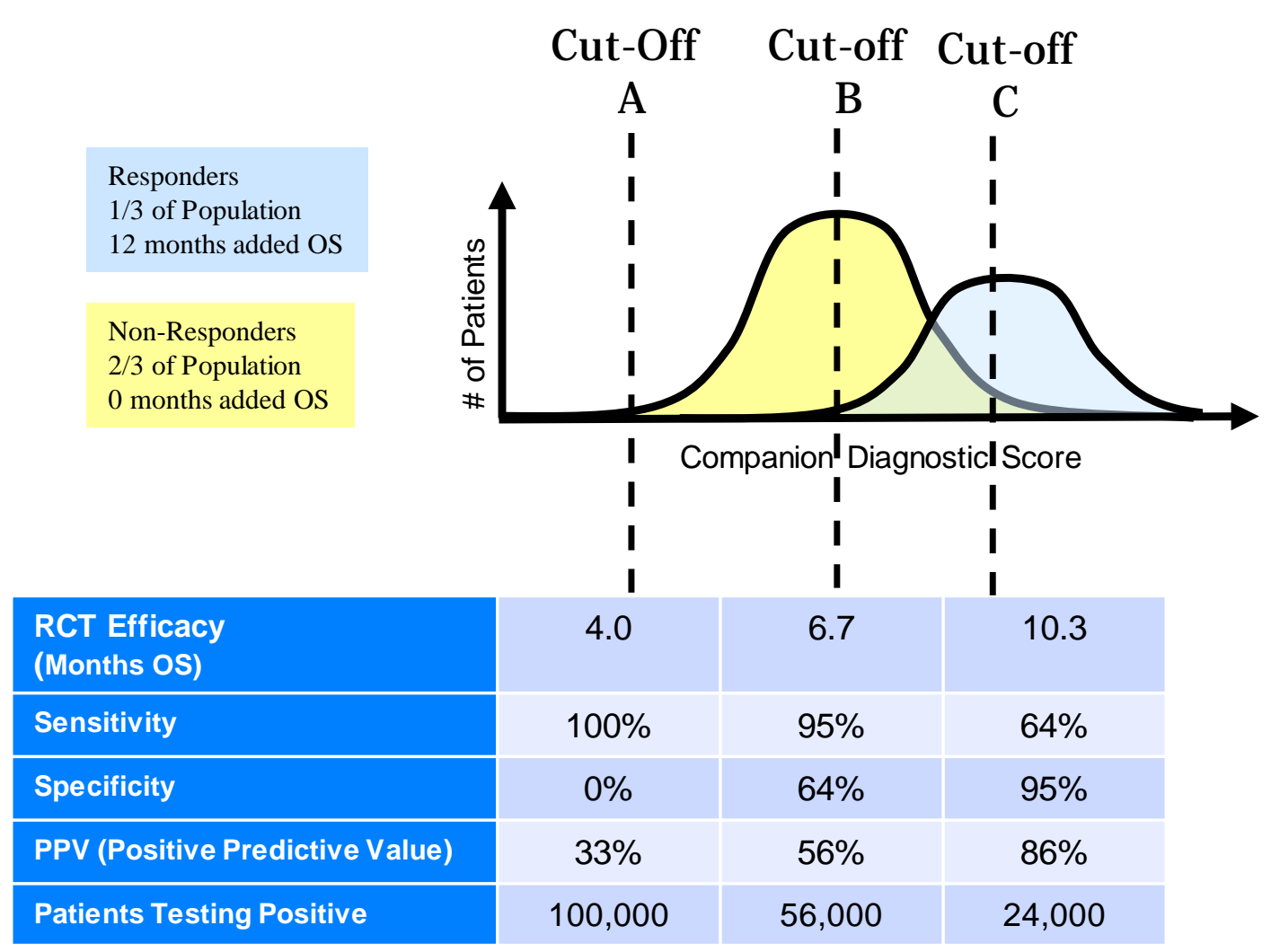

RCT: Randomized Controlled Trial OS: Overall Survival

Figure 1: Companion Diagnostics Affect Observed Efficacy. In this hypothetical case of an indication with 100,000 total patients, an imperfect companion diagnostic generally scores responders (blue) higher than non-responders (yellow) but with overlapping distributions. Cut-off A selects (those to the right of the cut-off) all patients. This is equivalent to an all-comers population with no diagnostic. Cutoff B selects nearly all patients who will respond ( $95 \%$ sensitivity) and raises the observed efficacy over $50 \%$ to 6.7 months by excluding non-responders. Cut-off C excludes nearly all non-responders $(95 \%$ specificity) which raises the observed efficacy to 10.3 months survival. See text for computational details.

Figure 1 illustrates the mechanics of how companion diagnostics achieve the biomarker science to clinical efficacy linkage. In the chart, the two curves represent all the patients with the disease who might be treated with the drug. The larger yellow curve to the left represents the patients who will not respond to the therapeutic. The smaller blue curve to the right 
represents those patients who will respond. The companion diagnostic test score along the $x-$ axis imperfectly separates the two patient populations with the vertical dashed lines indicating three different cut-off values for the test. Those to the right of the dashed cut-off line will be selected for treatment and those to the left will not.

The population overlap from the imperfect biomarker leads to patients with false positive and false negative test results. False positives are those patients represented by the portion of the yellow curve to right of the vertical cut-off line. False negative patients are those patients represented by the portion of the blue curve to the left of the cut-off.

To keep the mathematics simple, we assume that $33 \%$ of the 100,000 patients with this condition respond to treatment and that each responder gains 12 months overall survival compared to standard of care, and that the remaining patients receive zero incremental benefit. We also assume each distribution is standard normal and that the means are separated by two standard deviations. At cut-off A the clinical trial enrolls all patients to obtain an average clinical benefit of 4 months overall survival - the weighted average of the $1 / 3$ of patients who respond with the $2 / 3$ of patients who do not. Cut-off $A$ has $100 \%$ sensitivity (it selects all patients who might respond), and has $0 \%$ specificity (it excludes none who will not benefit). Another diagnostic metric, Positive Predictive Value (PPV) states the fraction of patients testing positive on the companion diagnostic $(C D x+)$ that actually do respond. More technically, PPV measures the number of true positives as a portion of all those who test positive. The all-comers PPV for cut-off $A$ is $33 \%$ - the responder prevalence rate. 
Using cut-off B selects nearly all who respond (95\% sensitivity, 31,500 of 33,000) but also includes many who do not. In our assumptions, cut-off B only yields $64 \%$ specificity so that 24,500 of 67,000 non-responders test positive (fall to the right of the cut-off B). For an oncology companion diagnostic this is quite superior performance. One of the more powerful companion diagnostics known, the KRAS test for detecting likely responders and nonresponders to cetuximab (Erbitux) in colorectal cancer, has an estimated $75 \%$ sensitivity and $35 \%$ specificity. ${ }^{16}$ By enriching for responders, using the companion diagnostic with cut-off $B$ will elevate the observed efficacy in the clinical trial to 6.7 months incremental overall survival (31,500 with 12 months additional survival and 24,500 with 0 months additional benefit). This overall survival improvement is about $70 \%$ longer than if no companion diagnostic was used (2.7 months longer than 4.0 months from using cut-off A). $56 \%$ of the patients testing positive $(C D x+)$ from using cut-off B would be expected to respond-the positive predictive value. This is 23 percentage points greater than the $33 \%$ of treated patients responding in an unenriched population. Choosing different cut-off values yields apparently different efficacy outcomes for the same molecule.

Using cut-off C excludes nearly all who do not respond (95\% specificity, 63,500 of $67,000)$. This high cut-off also excludes some patients who would benefit from treatment as measured by a lower $64 \%$ sensitivity (only $\sim 21,000$ of the 33,000 potential responders test positive and are eligible for treatment). A clinical trial using cut-off $\mathrm{C}$ would be expected to show a mean treatment survival benefit of 10.3 months (21,000 with 12 months additional survival and 3,500 with 0 months additional benefit). This is more than 2.5 times greater than the 4.0 months additional survival expected from a clinical trial not using a companion 
diagnostic (cut-off A). Also, the cut-off $\mathrm{C}$ reported overall survival benefit likely will be $54 \%$ longer (3.6 months longer than 6.7 months) than that for a trial using cut-off B. The power of the high cut-off $\mathrm{C}$ is demonstrated by the resulting high PPV, $86 \%$ of patients testing positive $(20,640$ of 24,000$)$ would be expected to respond. This is 53 percentage points higher than the $33 \%$ response rate expected from cut-off $A$.

Note that the innate drug performance is unchanged in these three scenarios. The differences are driven by the imperfect biomarker creating choices regarding whether to use it at all and if it is employed, what cut-off to choose. This scientific choice underpins the rules of the game. Assuming that pricing is at least somewhat proportional to efficacy, the companion diagnostic also sets up the pay-offs of the game.

Balanced clinical development impact

A precision medicine approach holds the potential for smaller, faster and less expensive clinical development due to the higher anticipated therapeutic effect owing to companion diagnostic use. However, the approach also requires the development of the diagnostic and its associated risk of failure, more complex patient recruitment and possibly no savings in trial size due to the potential need to examine negative test result patients and the continuing need to develop an acceptably large patient safety database. For this discussion we assume that these two effects exactly off set each other and so do not affect the games.

\section{The Diagnostic Cut-Off Prisoners' Dilemma Part 1}

The first game-like strategic decision precision medicine developers face is whether to use a companion diagnostic. Figure 2 illustrates the base case, iso-payoff game facing 
developers built on the Figure 1 case facts above. Assuming the companion diagnostic performance and cut-offs as before plus a price determined by a recent meta-analysis of oncology ICERs (Incremental Cost Effectiveness Ratios) of \$138,000 per incremental year of overall survival, a developer could choose to have their drug perform as Drug A, Drug B or Drug C in Figure 2. ${ }^{17}$ This further assumes that the drug developer faces a small number of other firms advancing drugs that engage the same molecular target, and possess essentially identical other characteristics such as adverse event and toxicity profile, dosing form, pharmacokinetics, pharmacodynamics, manufacturing costs and the like.

In practice, several precision medicines provide examples of this game theoretic dilemma. The exciting immuno-oncology therapies that target the PD1-PDL1 receptor-ligand complex are one such example. On average $10-20 \%$ of patients respond with many of those with dramatic remission that endures for over a year. The $10-40 \%$ of patients who respond to pembrolizumab (Keytruda, Merck \& Co.) receive dramatically overall survival increases compared to standard of care (6-10 months median overall survival in non-small cell breast cancer compared to docetaxol with $15 \%$ higher stable long-term survival-65\% compared to 50\%). ${ }^{18}$ Merck \& Co. chose to employ a PD-L1 companion diagnostic assay that was a de minimus fraction of the therapeutic cost. A competing firm Bristol Myers-Squibb developed nivolumab (Opdivo) without a companion diagnostic. ${ }^{19}$ Initially, the BMS product outperformed the Merck \& Co. product in the marketplace, in part due to easier use from not needing a test. ${ }^{20}$ However, nivolumab subsequently failed a trial in NSCLC (Non-Small Cell Lung Cancer) whereas the Merck \& Co. product succeeded in a similar trial partly because the PD-L1 companion 
diagnostic improved the observed efficacy by enriching the trial with those more likely to respond to pembrolizumab. ${ }^{22}$

\section{Responders $1 / 3$ of Population 12 months added OS \\ Non-Responders 2/3 of Population 0 months added OS}

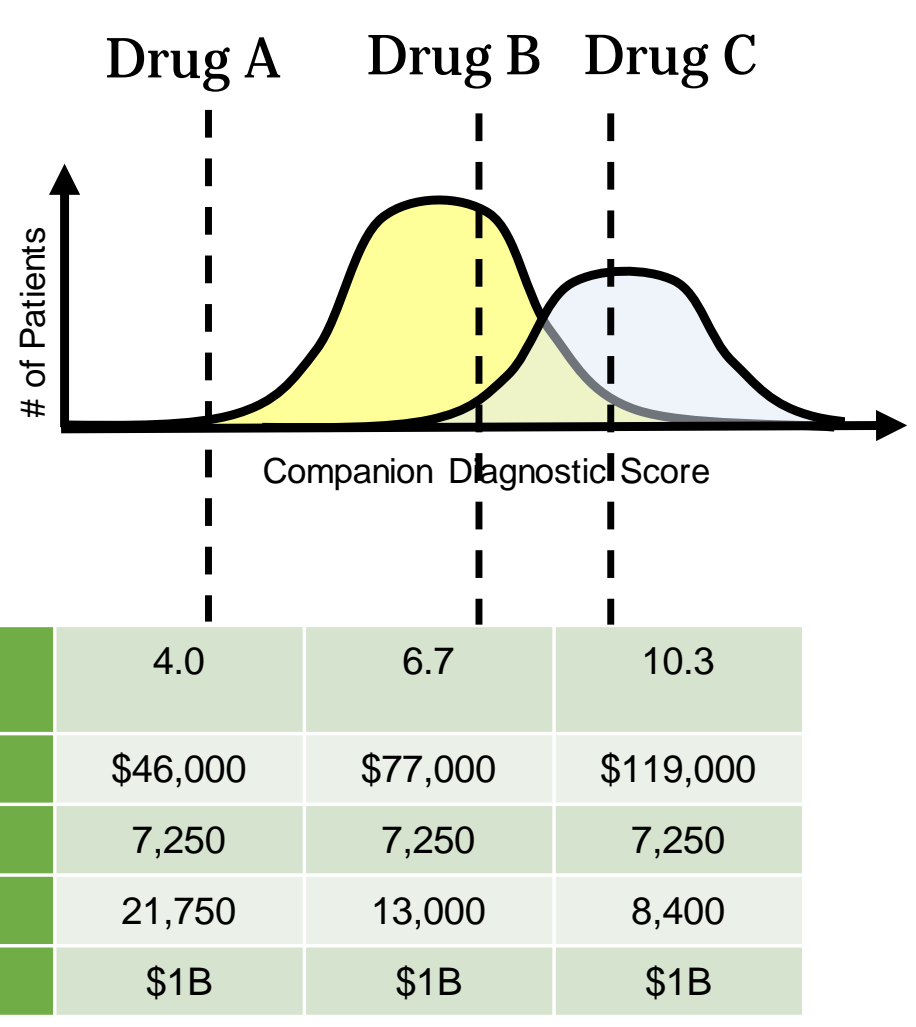

RCT: Randomized Controlled Trial OS: Overall Survival ICER: Incremental Cost Effectiveness Ratio

Figure 2: Companion Diagnostics Cut-Off Game Part 1. Continuing the Figure 1 hypothetical case of an indication with 100,000 total patients with an imperfect companion diagnostic, here we illustrate the potential drug prices based on a recent $\$ 138,000$ ICER oncology benchmark at the three Figure 1 illustrative companion diagnostic cut-offs. To reach $\$ 1 B$ of sales the no cut-off choice (Drug A) must treat 21,750 patients ( $21.75 \%$ market share in this 100,000 patient indication). Drug $B$ and Drug $C$ that each use a companion diagnostic must treat fewer total patients to achieve the same revenue and the same number of benefiting (responding) patients.

Other PD1-PDL1 immuno-oncology product developers have also faced the choice of whether to use a companion diagnostic test for their products such as atezolizumab (Tecentriq, Roche), avelumab (Bavencio, Pfizer, Merck KGaA), and durvalumab (Imfinzi, AstraZeneca). The 
firms developing atezolizumab and durvalumab both chose to use a PDL1 companion diagnostic but the avelumab developers did not. ${ }^{23}$ Those that chose to use a companion diagnostic not only chose different cut-off values but also chose to use distinct diagnostic tests with different performance characteristics. These choices have made it difficult to compare the clinical trial evidence among the products. Simultaneous development of the products hampered the ability to use consistent assays and so do not present a transparent 'game' to the drug developers. But the behavior also demonstrates that one strategy is to change the game through changing the test, not simply changing the cut-off value selection of a common test.

Note that like the classic prisoners' dilemma, each player would prefer a different outcome but each must choose a strategy to do the best they can, given the behaviors of the others. Thus the equilibrium iso-payoffs shown in Figure 2 suggest a strategy in which higher cut-offs might be preferred by each player.

\section{Anticipate a sequential game}

Seeing that cetuximab (Erbitux) had entered the market with a poor companion diagnostic (EGFR overexpression) that performed not much better than no CDx at all, the developers of panitumumab chose to use KRAS wild type status as to indicate non-response. ${ }^{24}$ This is similar to selecting the Drug $\mathrm{C}$ cut-off in Figure 2. This provided the panitumumab developers a short-term advantage until the developers of cetuximab could also show that their drug performed similarly with the KRAS marker. Due to relatively sticky prices, the cetuximab developers suffered revenue declines as patients were excluded but the price could not be raised to reflect the greater efficacy in the enriched patient sub-population..$^{25}$ Over time, 
however, cetuximab garnered increased revenues as more patients and physicians preferred cetuximab over panitumumab for other reasons (in the real world the assumption of identical other drug properties often does not hold) and annual price increases occurred for both drugs.

This example illustrates that developers must not only consider current players and diagnostics but also anticipate future players, new diagnostics and their impact on the precision medicine game.

Induced behavioral effects

Additional potential benefits not shown in Figure 2 may result from potential behavior changes induced by a precision medicine entering a market already served by other products. The perturbation from the new entrant will cause dynamic movements towards a new equilibrium (Figure 3). Although the first step to the new equilibrium reduces patient populations because of diagnostic exclusion, enhanced efficacy/safety increases market share as the therapy becomes the preferred treatment (Figure 3 from point $A$ to point $B$ to point $C$ ). If the companion diagnostic inspires greater confidence that the therapeutic is the best course for the patient, precision medicines may also benefit from improved patient adherence (C to D). Further market size and market share expansion occurs as underserved patients enter the market encouraged by the greater certainty of outcome should they qualify ( $D$ to $E$ ). This movement may be less pronounced in high mortality diseases such as oncology but more so for morbid conditions such as arthritis, HIV, Crohn's disease or psoriasis. Other factors may also encourage this shift. By providing higher, but not complete, assurance that the therapy will specifically work for them, the test shifts an individual patient's benefit odds and so helps overcome any barriers faced, from fear to inconvenience. A companion diagnostic may also 
encourage physicians to consider and recommend the therapy by prospectively indicating likely response. Even if the therapy is the only available treatment, a CDx might encourage providers and patients to initiate care.

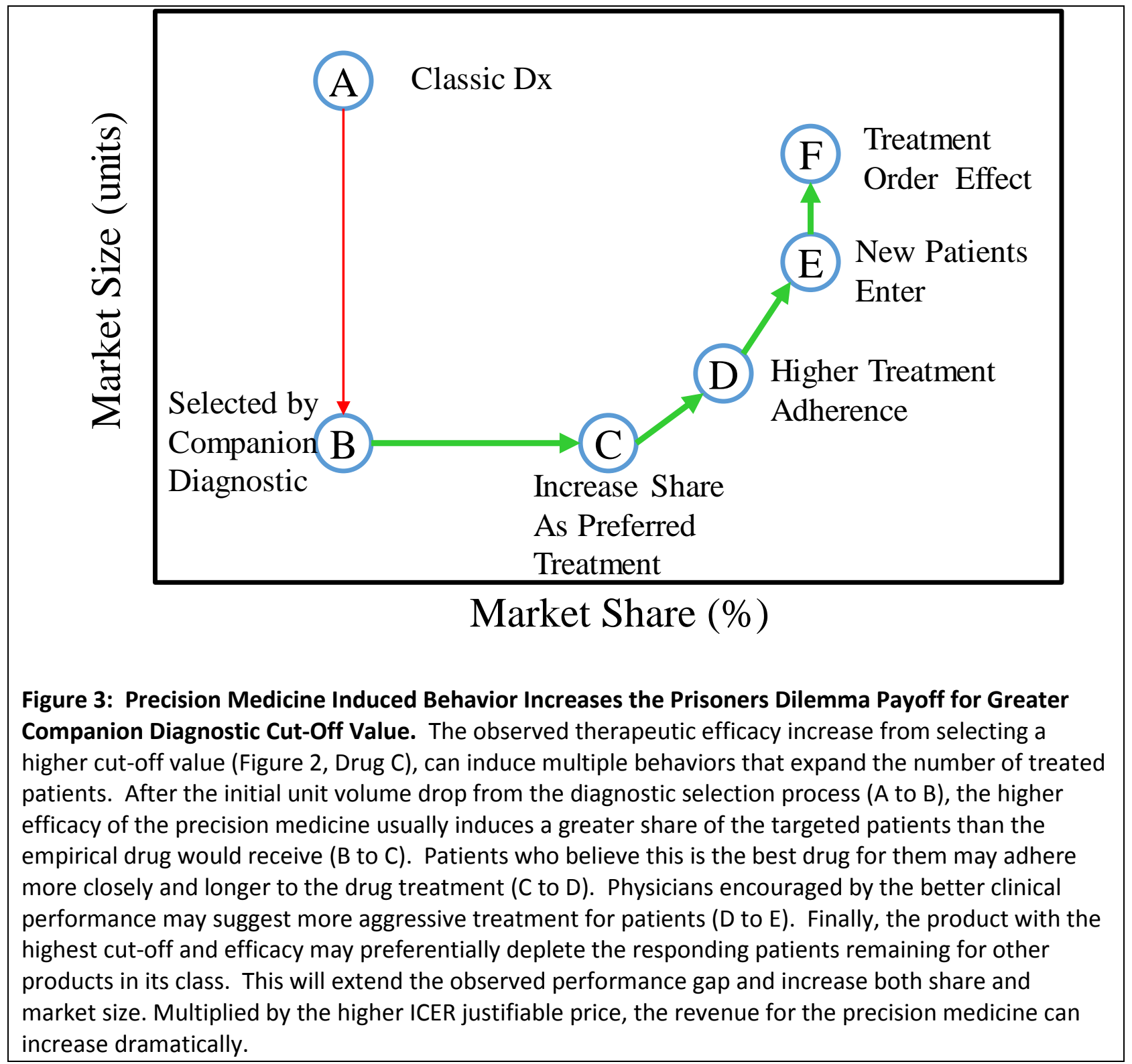




\section{Relaxing the Iso-Payoff Restriction}

The initial description in Figure 2 held the pay-offs as measured by payer cost and product sales constant for each cut-off selection. In this section, using just the increased market share induced behavior effect ( Figure $3 \mathrm{~B}$ to $\mathrm{C}$ ), we relax the iso-payoff assumption. In Figure 4, each strategy leads to a different market share in the selected population. Drug $A$ is hypothesized to now decline to $10 \%$ market share from $20 \%$ in the original Figure 2 case resulting in it treating 10,000 patients $(10 \%$ of 100,000$)$ and costing $\$ 0.5 \mathrm{~B}(\$ 46,000$ price times $10,000$ patients rounded to the nearest $\$ 0.1 B)$. Drug B is hypothesized to achieve $30 \%$ market share in its selected population of 56,000 test positive patients. Drug B therefore treats 16,800 patients $(30 \% * 56,000)$ and generates revenues of, or costs payers depending on one's perspective, $\$ 1.3 \mathrm{~B}(16,800$ patients times $\$ 77,000$ again rounded to nearest $\$ 0.1 \mathrm{~B})$. Drug $\mathrm{C}$ is hypothesized to achieve $55 \%$ market share in its selected population, 25 percentage points above the $30 \%$ market share hypothesized for Drug B. We calculate this hypothesized market share building upon the hypothesized Drug B $20 \%$ market share advantage (30\% vs. 10\%) over Drug A combined with the Drug B 2.7 months overall survival advantage (6.7 vs. 4.0 months) over Drug A. Drug C achieves a 3.6 month overall survival advantage (10.3 vs. 6.7 months) over Drug B. Extrapolating the same overall survival advantage to market share ratio yields a $26 \%$ additional Drug C market share which we round to 55\%. (20\% Drug B over Drug A market share increase $* 3.6 / 2.7=26 \%$ ). With a $55 \%$ market share Drug $C$ then generates $\$ 1.6 \mathrm{~B}$ in revenue (or payer cost depending on one's perspective) by treating 13,200 patients $(55 \% * 24,000 \mathrm{CDx}+$ patients) at a price per patient of $\$ 119,000$. 
Figure 4 illustrates the resulting prisoners' dilemma for both the firms and society. For illustrative purposes we assume that an individual firm wishes to maximize its firm profits by maximizing revenues. The high Drug C cut-off and corresponding pay-off maximizes the individual firm's revenues, and so profits, if it is the only firm to so choose. If all firms move to this high cut-off however, the overall collection of firms (assuming that $100 \%$ of patients would then receive treatment) will only receive $\$ 2.9 \mathrm{~B}$ in revenue with many potential responding patients not qualifying for treatment (those responders to the left of the Drug C cut-off in Figure 4).

Society would most benefit by treating the most responders while minimizing the treatment of non-responders. Given the imperfect companion diagnostic, this approximately corresponds to the Drug B cut-off which selects $95 \%$ of responders. The collection of firms also would be better off if the middle cut-off were selected. The Drug B cut-off would result in total market revenues (if $100 \%$ of those 56,000 testing positive were treated) of $\$ 4.4 \mathrm{~B}$ with 31,500 of $33,000(95 \%)$ potential responders benefiting. The middle cut-off also avoids potential adverse events and non-drug treatment costs for the 42,500 non-responders who would test negative and avoid ineffective treatment. 
Responders

$1 / 3$ of Population

12 months added OS

Non-Responders 2/3 of Population 0 months added OS

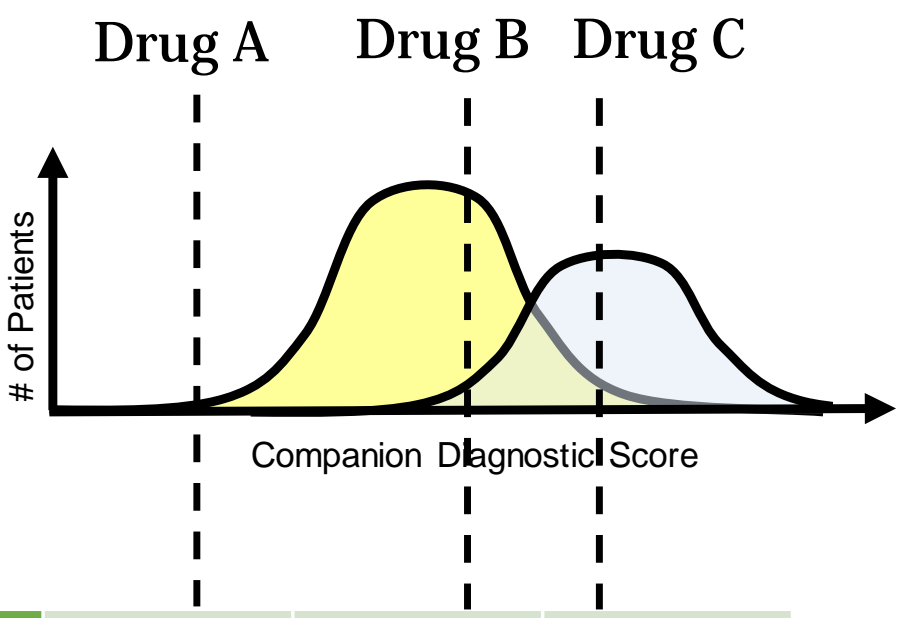

\section{RCT Efficacy \\ (Months OS)}

\section{Price (ICER Based)}

\section{Patients Testing Positive}

\section{Market Share in Selected}

\section{Patients Treated}

Product Revenue (Payer Cost)

Total $(100 \%)$ Market
4.0

$\$ 46,000$

100,000

$10 \%$

10,000

$\$ 0.5 B$

$\$ 4.6 \mathrm{~B}$
6.7

$\$ 77,000$

56,000

$30 \%$

16,800

\$1.3B

$\$ 4.4 \mathrm{~B}$
10.3

$\$ 119,000$

24,000

$55 \%$

13,200

$\$ 1.6 \mathrm{~B}$

$\$ 2.9 B$

ICER: Incremental Cost Effectiveness Ratio OS: Overall Survival

Figure 4: Companion Diagnostics Cut-Off Game Part 2: Varying Financial Pay-offs based on cut-off selection. Continuing the Figure 1 hypothetical case of an indication with 100,000 total patients with an imperfect companion diagnostic, here we illustrate the potential drug revenue (payer cost) pay-offs based on varying the market share in the selected population due to the induced behavior effect of perceived better efficacy (Figure $3 \mathrm{~B}$ to $\mathrm{C}$ ). Market shares in each selected population are hypothesized as $10 \%$ for Drug A, 30\% for Drug B and 55\% for Drug C. These shares of the respective test positive patient pools times the ICER based price create expected payer costs (developer revenue) that increases with the higher cut-off values. This creates a firm and social prisoners' dilemma because the high cut-off excludes patients who might benefit and with ICER based pricing if $100 \%$ of eligible patients were treated, cut-off B would generate both the most patient benefit and the most firm revenues $\$ 4.4 \mathrm{~B}$ revenue for 31,500 benefiting patients versus $\$ 2.9 \mathrm{~B}$ revenue and 20,700 benefiting patients for $100 \%$ market share at the highest cut-off) 


\section{Loser losses increased by responder depletion}

There might be additional market growth beyond that from the induced behavioral effects in the previous section (B to E in Figure 3). In clinical practice, Firm A might find it difficult to market and compete versus Firm B due to the difference in expected patient overall survival from the use of the companion diagnostic. From a public health perspective, an ordered market with selection bias in which more patients choose Drug B with the companion diagnostic could make the realized benefits from Drug A even lower. Since both work on the same biological target, initial use of Drug B may result in a residual Drug A patient subpopulation that is responder depleted. Thus rather than being used in an all-comers population with 33\% responders, Drug A may be relegated to treating a residual all-comers population with lower proportion of responders if Drug B achieves higher levels of market share among its companion diagnostic positive population.

If such therapeutic ordering occurs, real-world payer studies may therefore report Drug A efficacy substantially below the already lower average benefit observed in Drug A clinical trials whereas Drug B real-world studies would align with its original clinical trial observation. 
Responders

$1 / 3$ of Population

12 months added OS

Non-Responders 2/3 of Population 0 months added OS

\section{Drug A Drug B Drug C}

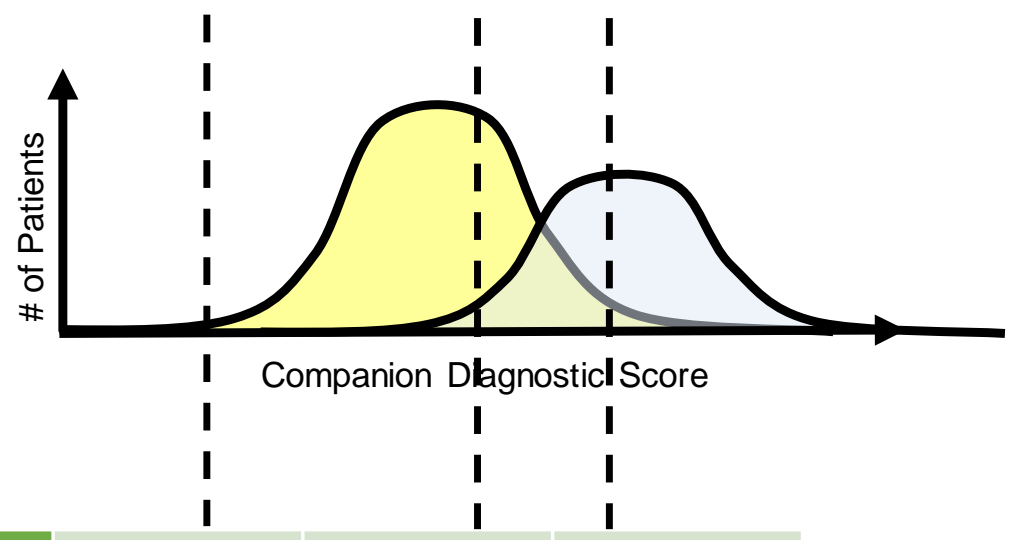

\section{RCT Efficacy \\ (Months OS)}

\section{Price (ICER Based)}

\section{Benefiting Patients}

Treated Patients

Payer Cost
0.4 (4.0 prior) $\$ 46,000$ 1,500 44,000 $\$ 2 B$
4.0 (6.7 prior)
10.3

$\$ 77,000$

$\$ 119,000$

10,800

20,700

24,000

$\$ 2.9 B$

ICER: Incremental Cost Effectiveness Ratio OS: Overall Survival

Figure 5 Responder Depletion Effect Increases Incentive to Select Higher Cut-off. If patients are first treated with Drug $C$, the pool of patients remaining for Drug $B$ that test positive have fewer responders. The population that remains is not similar to the clinical trial but to the population between the Drug $B$ and Drug $C$ cut-offs. In the real world this step therapy depletion effect would be expected to reduce the observed overall survival benefit for Drug B to 4.0 months from 6.7 previously and for Drug A to lower the OS survival benefit to less than half a month from the clinical trial result of 4 months.

\section{Game Theory Suggested Strategy}

The induced behavior phenomena seen in Figure 3 along with the responder depletion effect just discussed transform a simple one period game theoretic dilemma into a sequential one. Facing such a game, what strategy should a rational, game theory oligopolist adopt? While not game theorist specialists, we suggest that the optimal strategy is not clear. If one believes in efficacy proportional pricing (aka "value pricing"), we suggest that the optimal initial strategy 
may be to select a high biomarker cut-off to set an initial high price and protect against subsequent players selecting an even higher cut-off that would suggest that their product could make a superiority claim. Trastuzumab (Herceptin) effectively pursued this strategy with an initially high over-expression cut-off of $3+$ on the HER2 test. Over time, this cut-off has been lowered, at least unofficially, to include more potential responding patients. Cetuximab (Erbitux) is an example where not pursuing this strategy had negative results, at least for a period. ${ }^{16}$ Thus empirical support exists for the proposed initial high cut-off strategy.

This strategy is defeated, however, if one or more of the above assumptions do not hold. The immuno-oncology checkpoint inhibitors provide an example demonstrating the realworld risk. Bristol-Myers Squibb chose cut-off A (no PDL1 companion diagnostic) for nivolumab (Opdivo) while Merck \& Co. instead chose to use a companion diagnostic for its highly similar pembrolizumab (Keytruda). Companion diagnostics for checkpoint inhibitors are more cumbersome than other precision medicine genetic tests. Oncologists have preferred the ease and speed of choosing nivolumab over testing and waiting for pembrolizumab as evidenced by Bristol-Myers Squibb's product's sales being double that of the Merck \& Co product. In the sequential game where Merck \& Co. has now received positive non-small cell cancer first line treatment clinical trial results while Bristo-Myers Squibb has not, the dynamic may now be changing. But in the initial game theoretic round, not using precision medicine proved the superior choice for the drug developer. It is not clear this resulted in a patient optimal treatment outcome. 


\section{Variations of the Precision Medicine Prisoners' Dilemma}

Precision medicine science creates additional variants of the prisoners' dilemma game beyond that created by companion diagnostic enrichment. Here we describe two: precision medicine treatment dosing rules, and stopping criteria.

\section{The Dosing Game}

The FDA lists many drugs that have pharmacogenomic biomarkers (genetic tests) to indicate metabolism issues that can change the effective dose for either efficacy enhancement or adverse event avoidance. These biomarkers mostly involve liver enzyme mutations (CYP gene family and others). ${ }^{26}$ For example, mutations in the CYP2D6 gene in the cytochrome P450 family have been associated with metabolism differences for drugs as varied as the tricyclic antidepressants and selective serotonin reuptake inhibitors (SSRIs) and cardiovascular drugs such as beta blockers and antiarrhythmics. ${ }^{27}$ Mutations in another member of the gene family, CYP2C9, affect the metabolism of proton pump inhibitors, benzodiazepines, barbiturates and also the tricyclic antidepressants. Some mutations accelerate drug metabolism requiring increased dosing strength to maintain therapeutic levels in the blood. Other mutations slow drug metabolism requiring lower dosing strength to avoid effectively over-dosing patients. If their dose strengths were adjusted patients would see their beneficial responses rise and adverse events fall. The effect on drug revenues is more ambiguous because in some instances, assuming revenues are proportional to dosing strength, drug consumption would be increased, and so sales. In other instances, drug consumption would be lowered with drug revenues falling accordingly. In addition, as with companion diagnostics, improved dosing may induce 
second order behaviors. In this case, patient adherence may increase with concomitant increases in total drug usage and sales over time.

Developers thus face a variation of the prisoners' dilemma. Should they invest in elucidating the gene mutation effects and promoting the dosing changes in the hopes of improving patient care, gaining competitive advantage and increasing sales? Will competitors match their investments and so eliminate any preferential revenue payoff but raise costs for all? Or should the developer save the genetic discovery investment and hope others do as well? This game has an added feature: In clinical practice to date, physicians rarely test for these genetic mutations or use the test results to adjust their prescribed dosing. Unless physician practices change, the dosing game remains substantially moot. Or put another way, the genetic investment costs far outweigh the potential increased payoff-making the clearly dominant strategy to do nothing.

\section{The Stopping Game}

Precision medicine and diagnostic tests can indicate not just when a patient should begin a treatment but also when a patient would be best off to terminate a treatment. At least two sub-varieties of the stopping game have occurred. One suggests via a diagnostic that a therapy is not providing the expected benefit and so should be stopped. An example is the serum $M$ test for gauging the effectiveness of bortezomib (Velcade) from Johnson \& Johnson's Janssen for multiple myeloma after six weeks of treatment. ${ }^{28}$

The second stopping test sub-variety indicates when it is appropriate to halt a chronic therapy, at least for a time. Another, new therapy for multiple myeloma from Johnson \& 
Johnson's Janssen called daratumumab (Darzalex)/clonoSEQ is proving so effective that a new term is emerging, 'minimal residual disease', to indicate such dramatic remission that no cancer cells or their detritus can be detected. ${ }^{29}$ So called 'liquid biopsies' using next generation RNA and DNA sequencing tools on blood samples are being used to detect unique cancer cell signatures. When no signature is detected after general remission, the patient is declared to harbor minimal residual disease. Current studies are now ongoing to determine if chronic adjuvant therapeutic treatment can also be halted and then resumed only as necessary as indicated by a liquid biopsy conducted as a routine monitoring test.

Deciding whether to sponsor the trials for such a stopping rule creates a game theoretic dilemma for the drug company. Under a successful trial, drug revenues from chronic treatment decrease but could be offset by increases from patients preferring the treatment due to the high implied efficacy making stopping a possibility. Patients may also prefer the treatment owing to the possible savings and avoided adverse event risk from stopping. Like the companion diagnostic enrichment game, the payoffs may change as drug competitors facing the same choice do or do not also seek stopping rule tests. ${ }^{1}$

\section{Prisoner's Dilemma Part 3: Including The Payer}

The classic prisoners' dilemma focuses on the prisoner payoffs but usually ignores the jailor's desired information payoff from one or more prisoners and society's preference for seeing justice served. Including the payer perspective as another player but with a different

\footnotetext{
${ }^{1}$ The stopping rule presents a second game to the patient at the time when the stopping criteria have been met. Should the patient actually choose to halt therapy with the risk of more rapid cancer recurrence and benefit of no more drug cost, associated doctor visits and adverse events? Or should the patient continue treatment to keep the cancer at bay? The evidence supporting the stopping rule will likely be limited which will add uncertainty regarding the pay-offs in this game in which the patient literally bets their life.
} 
objective - to minimize the product revenue (i.e., minimize payer's costs) rather than maximize it - changes the rules of the game and introduces new payoffs for the payer. As Cortez demonstrated when he burned his ships, changing the rules can dramatically change payoffs and thus the player behaviors. In the case of Cortez, it removed the payoff of returning home for the conquistadors and so focused them on searching for gold in the New World - Cortez' preferred payoff. Introducing the payer as player not only transforms the payoffs but also moves the form of the game towards a Bertrand competition-the payer's preferred game.

A savvy payer might recognize that Drugs $A, B$ and $C$ in the example above are essentially identical but their developers use different companion diagnostic cut-offs to create the (inaccurate) perception of differentiated products. Faced with the table in Figure 2 above, such a savvy payer may decide to play to optimize its own pay offs (minimize its costs), rather than passively fund the drug company payoffs. 
Responders

$33 \%$ of Population

12 months added OS

Non-Responders $67 \%$ of Population

0 months added OS

\section{RCT Efficacy \\ (Months OS)}

\section{Price (ICER Based)}

Benefiting Patients

Treated Patients

Original Payer Cost

Revised Payer Cost

Savings

\section{Drug A Drug B Drug C}

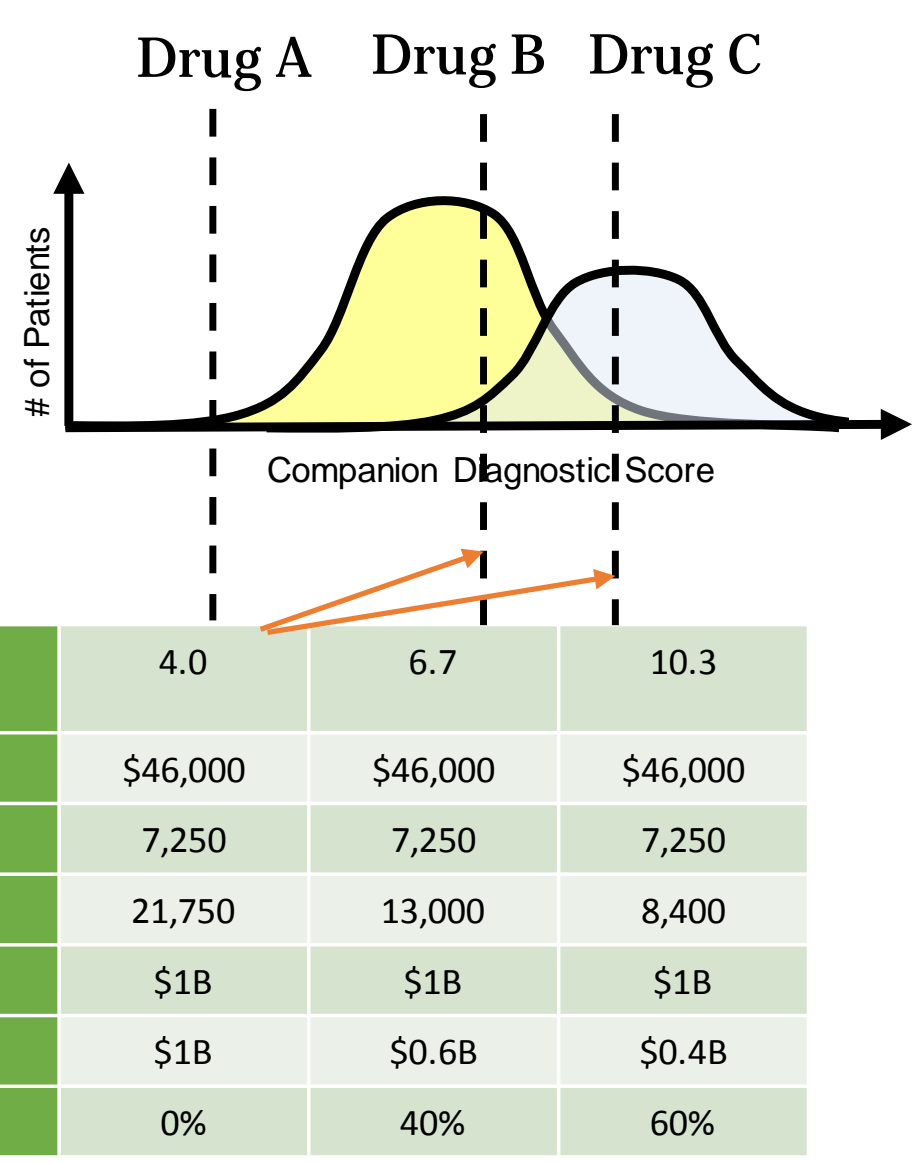

Figure 6: Companion Diagnostics Cut-Off Game Part 3: Adding the Payer as Player. Recognizing the fundamental equivalence of the three drugs differentiated by their companion diagnostic strategies, a savvy payer may switch Drug A for Drug B or Drug C. Alternatively, the payer may negotiate the lower price for Drug B or Drug $C$. The savings could reach $60 \%$ in this example.

Specifically, the payer might choose to use the cut-off from Drug C (the high cut-off) but require the use of Drug A (or negotiate discounts with Firms B and C to match Drug A pricing). Such a strategy would save the payer 60\% compared to purchasing Drug $\mathrm{C}$ as per Figure 2 . Because the Drug C cut-off excludes many who might benefit from treatment, the payer may choose the cutoff from Drug B to reach nearly all responders but with Drug A pricing. This would lower the total cost to $\$ 2.6 \mathrm{~B}$, while achieving the nearly perfect health benefit of about 32,000 QALYs/year. The effective cost per QALY correspondingly falls to $\$ 81,000$ from the $\$ 138,000$ reference used in these examples. This $40 \%$ price reduction from Drug B's price to 
Drug $\mathrm{A}^{\prime} \mathrm{s}$ price saves the payer $\$ 0.4 \mathrm{~B}$ of the $\$ 1.0 \mathrm{~B}$ initial illustration and $\$ 1.7 \mathrm{~B}$ compared to the \$4.4B amount the payer would expend at the ICER- justified Drug B price to treat $100 \%$ of companion diagnostic selected population. Such payer actions would of course reduce incentives for developers to pursue precision medicines in the future if in the end, they still only receive the all-comers non-stratified price.

This has a strong resemblance to finitely repeated prisoners' dilemmas in which backward induction causes all players to undercut from the first round onwards. ${ }^{30}$

Note that the payer has effectively transformed a precision medicine prisoners' dilemma game into a Bertrand competition for undifferentiated products.

\section{A Precision Medicine Bertrand Competition}

Generic, small molecule drugs, are certified by the FDA to be fully interchangeable and undifferentiated from each other and from the original branded product. In such an environment, payers and manufacturers engage in Bertrand competition, with the winning bidder (the lowest price bidder) potentially supplying the entire market. This competitive bidding process among undifferentiated products results in the price approaching marginal cost, since each bidder realizes bidding a price above marginal costs risks being underbid by a competitor. When products are highly substitutable but not identical, payers may still possess significant pricing power which they may exercise in Bertrand competition like behaviors. ${ }^{31}$ For example, CDC used to have winner take all bidding for vaccines which successfully lowered prices to the lowest marginal cost of the lowest cost manufacturer which drove all others out of the market. ${ }^{32}$ In the face of this Bertrand Competition result, the CDC altered its bidding 
process by allowing individual states to run bidding competitions with evaluation criteria beyond lowest price. The number of vaccine manufacturers has since rebounded to restore both competition and supply security. ${ }^{33}$

Precision medicines can also experience situations very similar to Bertrand competition. The new generation of Hepatitis $\mathrm{C}$ treatments are precision medicines. Hepatitis $\mathrm{C}$ infections are classified into six major genotypes or strains creatively named 1 through 6 . Perhaps the most famous Hepatitis C treatments are sofosbuvir (Sovaldi) and the fixed-dose combination of ledipasvir-sofosbuvir (Harvoni) from Gilead Pharmaceuticals. Sovaldi essentially cures patients with genotypes 1 through 4 when combined with other supporting drugs, while Harvoni was initially approved as a sole treatment for patients with genotypes 1 or $4 .{ }^{34}$ Harvoni has subsequently been approved to also treat patients with Hepatitis $C$ genotypes 5 and 6 . Thus, to select the appropriate treatment the patient must first have their infection genetically identified with an immunoassay blood test which detects the genotype specific antibodies the patient's body produces to combat the virus. ${ }^{35}$ Sovaldi was the first drug launched in its new class of chemistry that both attacks the unique Hepatitis C NS5B protein (critical for its machinery of replicating inside the human cell) and that efficiently penetrates the host human cell.

Sovaldi was launched in December 2013 with a list price of $\$ 84,000$ per patient course of treatment. This pricing received widespread publicity and incurred considerable criticism from payers and those who sympathized with them. Harvoni launched soon after with a list price of $\$ 94,500$ for its one tablet a day, 12-week treatment course. Gilead justified these prices based on the value to the healthcare system for avoiding the expensive liver transplants and 
other care that advanced Hepatitis $C$ patients incur, and by Harvoni being priced at rough parity per cure obtained compared to earlier generations of hepatitis $C$ treatments.

Gilead only had the market to itself for one year. In early December 2014 AbbVie received approval for Viekira Pak which combined three drugs to cure those patients with the most prevalent Hepatitis C genotype, genotype 1. By Christmas, Express Scripts (at the time the largest pharmacy benefits manager in the United States) announced that its National Preferred Formulary would exclude Gilead's Sovaldi and exclusively offer AbbVie's Viekira Pak which it claimed it received at a large discount. ${ }^{38}$

Since then, in August 2017 AbbVie has launched a new drug Mavyret that cures in only eight weeks rather than the 10-14 weeks required of the prior drugs and at a list price of $\$ 26,400$. The National Acquisition Center makes available the federal government pharmaceutical purchasing cost in the four major programs of the Federal Service System (FSS) and the so-called Big 4 (DoD, VA, Public Health Service and Coast Guard) prices. The lowest shown prices of Sovaldi, Harvoni, Viekira Pak and Mavyret at the end of August, 2017 extrapolated for a full treatment course are $\$ 49,860, \$ 56,700, \$ 60,153$ and $\$ 29,235$ respectively. ${ }^{39}$ The gradual decline to something closer to the Bertrand Competition marginal cost continues with price discounts and rebates, some of which are government mandated.

\section{Defending Against a Bertrand Competition: Biologics}

A game with payers included can be transformed into Bertrand competition as discussed above for small molecules, but in large molecule biologics competition may be limited by the entry deterring science of proving similarity and interchangeability. To date, to protect patient 
safety, the FDA has certified some biologics to be biosimilar but not interchangeable, thereby not fully enabling Bertrand competition. ${ }^{40}$ In Europe biologics have been successfully defending their franchises for nearly a decade. ${ }^{40}$ Janssen's Remicade is successfully defending its brand position in the US against biosimilars such as Inflectra from Pfizer. ${ }^{41}$ Janssen appears to be pursuing a three pronged strategy of payer negotiations with increased rebates to secure exclusive first line contracts; volume based discounts with providers to incentivize stocking; and bundling Remicade with other Johnson \& Johnson products to reduce Inflectra appeal as a standalone discount. Janssen has pursued a discounting approach that leverages its economies of scale and scope and lingering medical concerns that inhibit switching all patients to a biosimilar thus requiring some continued inclusion of Remicade by providers and payers. In short, sophisticated discounting and continued product differentiation has blunted the price advantage of the Inflectra biosimilar.

Game theory models with learning formalize these defense strategies, but we do not have space to pursue them here. ${ }^{42}$

\section{The Emerging Multi-Class Precision Medicine Game}

We have described some of the games that cohorts of precision medicines face within their niches. While each niche is defined by its scientific therapeutic target and the associated biomarkers, the niches may begin to overlap and compete for the same patients. Such a situation is emerging in inflammatory disease where no fewer than five drug classes will be competing for rheumatoid arthritis, Crohn's disease and psoriasis patients. Each of these drug classes provides significant benefits for as few as $30 \%$ or as many as $60 \%$ or maybe even $75 \%$ of patients. Biomarkers exist or are being explored for many of these drug classes. 
Drug developers, payers and patients face a complex simultaneous game in these multiclass situations. This is built upon, but significantly different from, the multi-player within a product class games we discussed above. Each precision medicine niche will likely be at a different maturity stage. Some will be early in their lifecycles while others will be entering postexclusivity. The games will likely link, with the payoffs of the early lifecycle games being influenced and constrained by the payoffs and strategies employed by the later games. Similarly, the late stage game will be influenced by the new niches which may threaten them with technological obsolescence.

The five set Venn diagram in Figure 7 shows the combinations and competition emerging in these overlapping precision medicine niches. The legend assigns a current or emerging inflammatory drug class to each set, A through E. Each class may provide benefits exclusively to some patients, represented as the pure, non-overlapping color at the exposed point of each set. All other products fail for these patients. Most patients however will likely respond to two, three, four or maybe all the drug classes. These are represented by all the overlapping, labelled sections. Note that the schematic shows the logical overlap but does not necessarily show the quantitative actual results. Some small section(s) on the schematic may contain large numbers of patients and vice versa. Also, the schematic does not show the degree to which patients respond more strongly or more weakly to the product classes within a multi-class intersecting area. Navigating the game and determining the optimal strategies for the players is beyond the scope of this paper and will of course depend upon the specific payoffs as determined by the performance of the products and patient value inherent to these indications. The developers, payers, providers and patients in this inflammatory disease space 
nonetheless are facing this complex game, whether they fully comprehend its complexity or not. We suggest that game theory can provide multiple insights to guide the clever, rational player.

$$
\begin{aligned}
& A=N K G 2 D \\
& B=T N F a \\
& C=I L-12, I L-23 \\
& D=a 4 \beta 7 \text { or aE } \beta 7 \\
& E=\text { Smad7, Orals (JAK,S1P1), Etc. }
\end{aligned}
$$

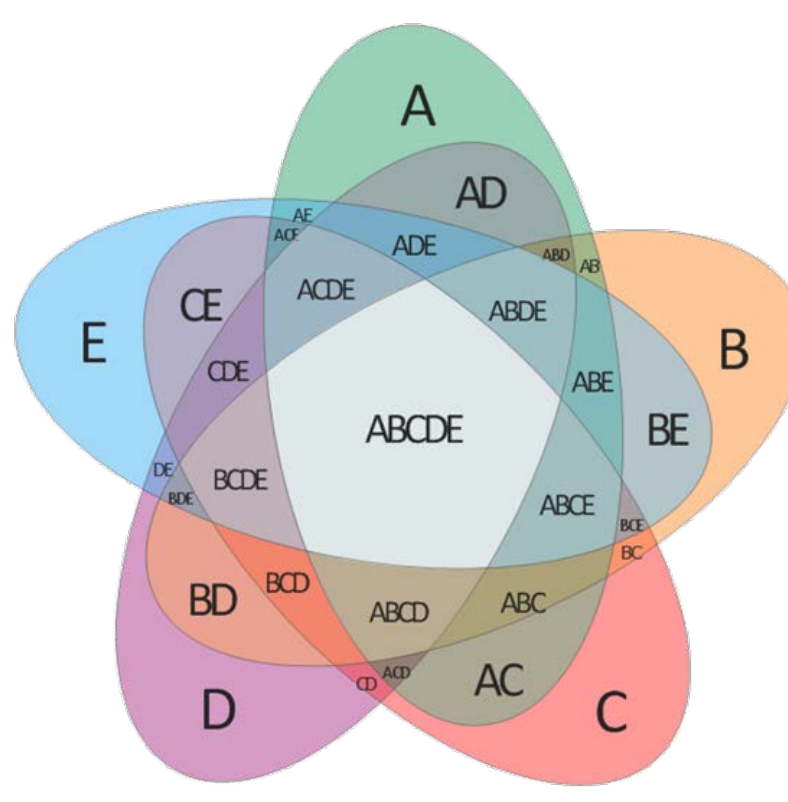

Figure 7: The emerging multi-class game for inflammatory diseases such as rheumatoid arthritis, irritable bowel disease, Crohn's disease and psoriasis, among others maybe even cardiovascular.

Oncology faces a similar explosion of product classes for an individual cancer type with the even more complex possibility of including drug combinations to increase efficacy for some molecularly defined patient sub-populations. Oncology has maintained stronger differentiation of each molecular sub-type as a distinct market limited to a particular cohort of precision medicines. For example, HER2-positive breast cancer remains distinct from other precision medicines and classic medicines. This keeps the prisoners' dilemma game mostly intact for Roche and its competitors GlaxoSmithKline and Puma Biotechnology. Roche has two products in the game -- trastuzumab (Herceptin) and trastuzumab emtansine (Kadcyla, T-DM1 that adds 
a cytotoxic chemotherapeutic to the trastuzumab antibody for double action). The other players compete with differently targeted precision medicines that are also approved for HER2 positive breast cancer patients. GlaxoSmithKline markets lapatinib (Tykerb) and Puma Biotechnology offers neratinib (Nerlynx).

\section{The Phased Precision Medicine Game}

We have explored a series of precision medicine examples and the game situations they exemplify. These examples also suggest that cohorts of precision medicines that share niches might present a series of linked games over their lifecycles (Figure 8).

As the precision medicine cohort emerges, the drug developers face the first game theoretic dilemma as they make decisions regarding their clinical trial programs and prepare for regulatory submissions. It is likely the companion diagnostics core game but may be the dosing or stopping rule variants, or even a combination of the three.

After market authorization, and the entrance of multiple near substitute precision medicines, the payers may enter the game and attempt to change it into a Bertrand competition. This has been demonstrated in the Hepatitis $C$ market. Payers may learn to develop their own scientific insights to mix diagnostics with therapies as we suggested in the companion diagnostics game part 2 above. 


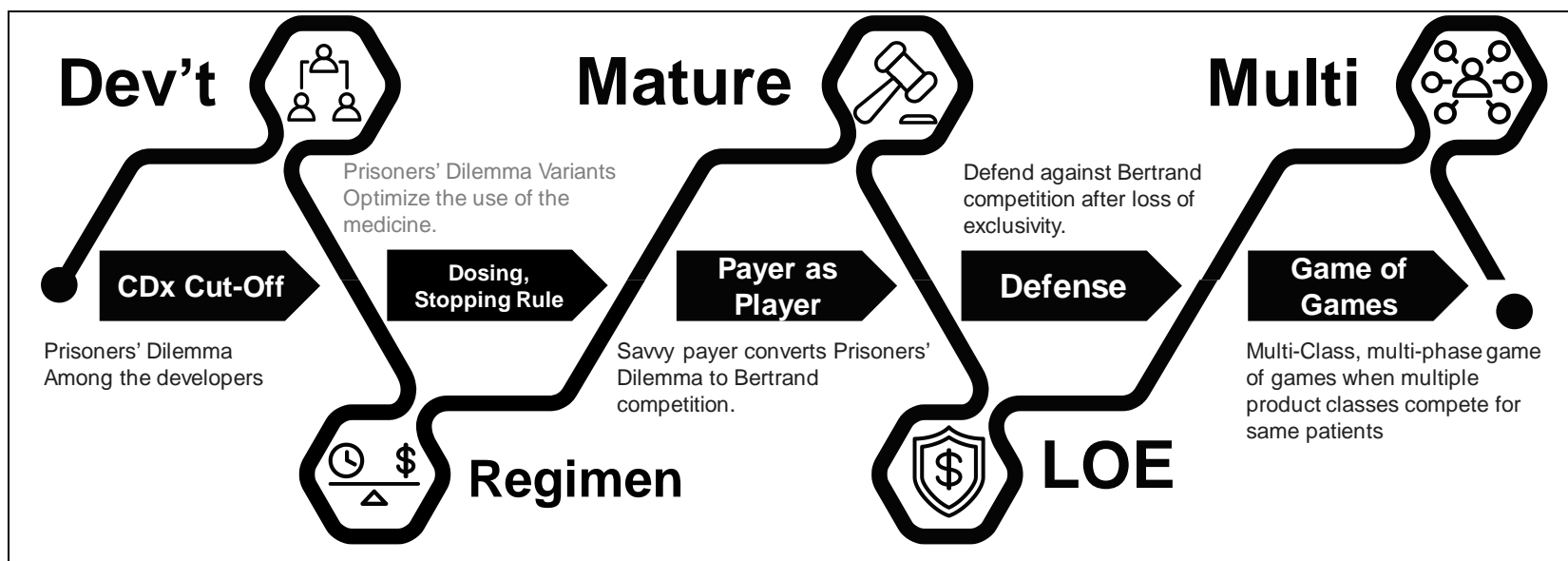

Figure 8: The Phased precision medicine lifecycle game. The games can be arrayed across the life cycle of a therapeutic. While not guaranteed to occur for all precision medicines, a developer might choose to prepare for the sequential, connected games that the individual specific cases suggest might occur.

If the branded therapeutic does not succumb to a Bertrand competition prior to legal loss of exclusivity, traditional biologics products are demonstrating an ability to deter entry and combat franchise erosion. Traditional small molecule brands have also demonstrated some ability to delay, but less ability to defeat, generic competition. Precision medicine remains too young to have examples of biosimilar or generic competition. But simply because it has not yet occurred does not mean that the generic/biosimilar defense game is unlikely when exclusivity ends for these products.

Participants in precision medicine markets can expect a series of games and opportunities to apply game theory for each market. Participants will need to discern, however, which game applies to each cohort or niche at a given time. Participants will also need strategies that link across games as payoffs from the prior games may influence later games, and so reputational effects may evolve. In any case, the preparation for later games will begin even as the initial games unfold. 


\section{Conclusion}

In this paper we have described how precision medicines create situations that bear some semblance to game theory constructs such as the prisoners' dilemma and Bertrand competition. We recognize that the textbook examples of the Prisoners' Dilemma are typically a one-time static game, whereas what we have described here is more dynamic in nature. But the resulting Nash equilibrium outcome is similar. In particular, outcome equilibria emerge in which players each prefer a different outcome but must choose a strategy to do the best they can assuming the anticipated rational behaviors of the others. The examples usually involve drug developers in competition with each other. We have also described situations in which other stakeholders join the game or face their own unique game. For instance, when payers joined the prisoners' dilemma game they transformed it into a Bertrand competition-like game. Another prisoners' dilemma-like game occurs when patients face their own distinct decision in the stopping rule variant. When patients join the drug developer companion diagnostic prisoners' dilemma, their induced behaviors amplifies the payoffs for the primary drug developer players.

We also observe that the games may predictably array themselves across the lifecycle of each precision medicine indication niche. These arrayed games may remain independent or may become linked into a sequentially evolving meta game. This might be an area for additional fruitful theoretical research immediately and for future empirical studies as the precision medicine niches mature. 
Finally, we hypothesize that some precision medicine areas such as inflammatory diseases are becoming complex simultaneous multi-games in which separate drug cohorts in distinct precision medicine niches compete with other cohorts for patients as well as within their own cohort. This multi-game is further complicated by cohorts being in different lifecycle stages even as the cohorts compete across niches. Some cohorts may be young and just emerging into the market, while others are established and still others may be entering the mature loss of exclusivity game. By stretching the classic game frameworks, this multi-game might be a particularly exciting, albeit challenging, area for future research.

Precision medicines have been a limited pharmaceutical category since their introduction in the late 1990s. Rapidly increasing molecular understanding of disease and the falling cost of genetic testing due to next generation sequencing technologies have swollen the precision medicine product pipeline in many therapeutic areas. Whether these precision medicines can achieve sustainable commercial success and payer acceptance remains to be seen. The underlying science may support virtually unlimited differentiation, but economic forces may advance commoditization, particularly if developers continue to introduce multiple products in each precision medicine scientific niche. Much will depend on how successfully developers play the prisoners' dilemma to differentiate their products even as payers attempt to transform that game into a Bertrand competition.

In such a context, the credibility of evidence sources will play a critical role, again raising enduring issues of the optimal societal roles for public and private sectors in creating, disseminating, and pricing information. Those players that learn the most rapidly and apply 
those learnings the most asymmetrically will be advantaged in this ongoing information pharms race. 


\section{REFERENCES}

1. Trusheim, Mark R., Ernst R. Berndt and Frank L. Douglas, "Stratified Medicine: Strategic and Economic Implications of Combining Drugs and Clinical Biomarkers", Nature Reviews: Drug Discovery, April 2007, 6(4):287-293.

2. Hu, Sean X., Murray L. Aitken, Arnold M. Epstein, Mark R. Trusheim and Ernst R. Berndt, "Defining and Quantifying the Use of Personalized Medicines", Nature Reviews: Drug Discovery, online version published 29 November 2013, http://www.nature.com/nrd/journal/v12/n12/full/nrd4177.html?WT.ec id=NRD-201312. Hard copy version published December 2013.

3. Novartis press release. Novartis CAR-T cell therapy CTL019 unanimously (10-0) recommended for approval by FDA advisory committee to treat pediatric, young adult $r / r$ B-cell ALL. July 13, 2017. https://www.novartis.com/news/media-releases/novartis-car-t-celltherapy-ctl019-unanimously-10-0-recommended-approval-fda , Accessed August 22, 2017.

4. Press Release. Sanpower Group closes acquisition of Dendreon. June 29, 2017. Available at http://www.dendreon.com/Portals/188/DENDREON\%20PRESS\%20RELEASE.pdf . Accessed August 22, 2017. OR Reuters staff. BRIEF-Valeant pharmaceuticals completes sale of Dendreon to Sanpower Group. Reuters. June 29, 2017. http://www.reuters.com/article/brief-valeantpharmaceuticals-completes-idUSFWN1JQ07C Accessed August 22,2017.

5. FDA. Cellular \& Gene Therapy Products-Marketed Products. Food and Drug Administration. https://www.fda.gov/BiologicsBloodVaccines/CellularGeneTherapyProducts/ApprovedProducts Ldefault.htm Page updated August 11, 2017. Accessed August 22, 2017.

6. Trusheim, Mark R., Breon Burgess, Sean Xinghua Hu, Theresa Long, Steven D. Averbuch, Aiden A. Flynn, Alfons Lieftucht et al. "Quantifying factors for the success of stratified medicine." Nature Reviews: Drug Discovery 10, no. 11 (2011): 817.

7. US Food and Drug Administration. Remicade (infliximab) label, revised: January 2015. US Food and Drug Administration. Available at: http://www.accessdata.fda.gov/scripts/cder/drugsatfda/ (2015).

8. Helwick, C. Pathologic complete response: Understanding the subtleties. The ASCO Post 5(16) October 15, 2014. accessed on 5/4/2015. Available at http://www.ascopost.com/issues/october-15,-2014/pathologic-complete-responseunderstanding-the-subtleties.aspx (2014).

9. Ainsworth R., et al. IHC for Her2 with CBE356 antibody is a more accurate predictor of Her2 gene amplification by FISH than HercepTest ${ }^{\mathrm{TM}}$ in breast carcinoma. J. Clin. Pathol. 58 1086-1090 (2005). 
10. Birner P., et al. Evaluation of the United States Food and Drug Administration-approved Scoring and Test System of HER-2 Protein Expression in Breast Cancer. Clin. Can. Res. 7 16691675 (2001).

11. Hu, Sean X., Murray L. Aitken, Arnold M. Epstein, Mark R. Trusheim and Ernst R. Berndt, "Defining and Quantifying the Use of Personalized Medicines", Nature Reviews: Drug Discovery, online version published 29 November 2013, http://www.nature.com/nrd/journal/v12/n12/full/nrd4177.html?WT.ec id=NRD-201312. Hard copy version published December 2013.

12. Trusheim, Mark R. and Ernst R. Berndt, "The Segmentation of Therapeutic Populations in Oncology", Health Management, Policy and Innovation, 1(1):19-34, October 2012

13. Trusheim, Mark R., and Ernst R. Berndt, "The clinical benefits, ethics, and economics of stratified medicine and companion diagnostics", Drug Discovery $\quad$ Today 20(12): 14391450, December 2015.

14. Robert S. Pindyck and Daniel L. Rubinfeld, "Game Theory and Competitive Strategy", ch. 13 in Pindyck and Rubinfeld, Microeconomics, Eighth Edition, New York: Pearson Education, Inc., publishing as Prentice Hall, 2013, 487-528.

15. FDA. FDA Online Label Repository, available at http://labels.fda.gov/ (2017).

16. Westwood M, van Asselt T, Ramaekers B, et al. Health Technology Assessment, No. 18.62. Chapter 3 Table 8 Accuracy of KRAS mutation testing for the prediction of response to treatment with cetuximab in addition to chemotherapy. Southampton (UK): NIHR Journals Library; 2014 Oct

17. Bae Y.H. Mullins C. D. Do value thresholds for oncology drugs differ from nononcology drugs? J. Manag Care Pharm, 20(11):1086-92. (Nov 2014).

18. FDA. Keytruda label. FDA Online Label Repository, available at http://labels.fda.gov/ (2017).

19. FDA. FDA Online Label Repository, available at http://labels.fda.gov/ (2017).

20. Staton, T. Bristol-Myers' Opdivo far outpaced Merck's Keytruda again in Q2. What gives? FiercePharma August 1, 2016. http://www.fiercepharma.com/marketing/bristol-myers-opdivo-far-outpacedmerck-s-keytruda-again-q2-what-gives Accessed August 27, 207.

21. Pollack, A. Immunotherapy drug Opdivo fails clinical trial to expand use. New York Times. August 5, 2016. https://www.nytimes.com/2016/08/06/business/lung-cancer-drug-opdivo-failsclinical-trial-to-expand-use.html?mcubz=1 Accessed August 27, 2017. 
22. FDA. FDA Online Label Repository, available at http://labels.fda.gov/ (2017).

23. FDA. FDA Online Label Repository, available at http://labels.fda.gov/ (2017).

24. FDA. FDA Online Label Repository, available at http://labels.fda.gov/ (2017).

25. Carlson B. KRAS testing: Optimizing Cancer Therapy. Biotechnoloyg Healthcare. 6(5):7-9 Winter 2009-2010.

26. FDA. Table of Pharmacogenomic Biomarkers in Drug Labelling. FDA. Available at

https://www.fda.gov/drugs/scienceresearch/researchareas/pharmacogenetics/uc m083378.htm. Accessed August 27, 2017.

27. Gardiner, S.J. and Begg, E.J. Pharmacogenetics, drug-metabolizing enzymes, and clinical practice. Pharmacological reviews, 58(3):521-590 (2006).

28. NICE. Bortezomib monotherapy for relapsed multiple myeloma. NICE technology appraisal guidance 129. Issue date October 2007. Review date October 2010. Available at https://www.nice.org.uk/guidance/ta129 Accessed August 27, 2017.

29. Press release. Adaptive Biotechnologies Announces a Collaboration with Janssen Biotech, Inc. to use the clonoSEQ ${ }^{\circledR}$ Assay to Measure Minimal Residual Disease in Ongoing and Future DARZALEX ${ }^{\circledR}$ Multiple Myeloma Trials. July 21, 2017. Available at http://www.adaptivebiotech.com/news/adaptive-biotechnologiesannounces-collaboration-janssen-biotech-inc-use-clonoseq\%C2\%AE-assay Accessed August 27, 2017.

30. Pindyck, RS, Rubinfeld DL. Microeconomics Eighth Edition. pp:499-500, Pearson Education, 2013.

31. Berndt ER, MCGuire TG, Newhouse JP. A primer on the economics of prescription pharmaceutical pricing in health insurance markets. Forum for Health Economics \& Policy 14(2) article 10, November 2011.

32. Danzon, Patricia M., Nuno Sousa Pereira, and Sapna S. Tejwani. "Vaccine supply: a cross-national perspective." Health Affairs 24, no. 3 (2005): 706-717.

33. Berndt, E.R., Denoncourt, R.N. and Warner, A.C. US markets for vaccines: characteristics, case studies, and controversies. AEI Press. (2009).

34. FDA. FDA Online Label Repository, available at http://labels.fda.gov/ (2017).

35. CDC. Hepatitis C Information on Testing and Diagnosis. Centers for Disease Control. Available at https://www.cdc.gov/hepatitis/hcv/pdfs/hepctestingdiagnosis.pdf , Accessed August 31, 2017. 
36. Pollack, A. FDA approves pill to treat Hepatitis C. New York Times, Page B1, New York Edition, December 7, 2013. Available at http://www.nytimes.com/2013/12/07/business/fda-approves-pill-to-treathepatitis-c.html?mcubz=1 . Accessed August 31, 2017.

37. FDA. FDA Online Label Repository, available at http://labels.fda.gov/ (2017).

38. Humer C. Express Scripts to cover AbbVie hepatitis $C$ drug, drops Gilead treatment. Reuters. December 22, 2014. Available at http://www.reuters.com/article/express-scripts-abbvie-hepatitiscidUSL1N0U50M120141222 Accessed August 31, 2017.

39. National Acquisition Center (CCST) Pharmaceutical Catalogue Search. US Department of Veterans Affairs. Available at https://www.va.gov/nac/Pharma/List, Accessed August 31, 2017.

40. Berndt, E.R. and Trusheim, M.R. Biosimilar and Biobetter Scenarios for the US and Europe: What Should We Expect?. In Biobetters (pp. 315-360). Springer New York. (2015).

41. Private communication with Aaron Gal of Berstein

42. Fudenberg, D. and Tirole, J., 1991. Game theory, chapter four. Cambridge, Massachusetts: MIT Press. 